\title{
The media campaign on the DSM-5: recurring comments and lessons for the future of diagnosis in psychiatric practice
}

\author{
M. Maj \\ Department of Psychiatry, University of Naples SUN, Naples, Italy
}

\begin{abstract}
Recurring arguments in the media campaign preceding and following the publication of the DSM- 5 have been that the manual, referred to as 'the bible of psychiatry', mislabels many people who are basically normal, and that the diagnostic categories it contains are invalid, not being based on laboratory tests. We present data on the use of the DSM worldwide, and discuss the need to assess systematically the pros and cons of operational and prototype approaches to psychiatric diagnosis. We consider different views about what qualifies as mental disorder and how the boundary between pathology and normality should be fixed. We review the role of laboratory tests as applied in medicine, emphasising that most of them are probabilistic, not pathognomonic, markers of disease. We finally summarise the promise and limitations of the Research Domain Criteria project, aiming to 'transform psychiatric diagnosis' by replacing descriptive psychopathology with behavioural and neurobiological measures.
\end{abstract}

Received 24 July 2014; Accepted 5 August 2014; First published online 10 September 2014

Key words: DSM-5, psychiatric diagnosis, mental disorder, laboratory tests, RDoC.

The recent publication of the DSM-5 has been preceded and followed by an avalanche of critical comments in the media, which have targeted not only the new edition of the manual, but also more in general the practice of psychiatric diagnosis.

Recurring statements have been that the DSM is 'the bible of psychiatry' (e.g., Horgan, 2013), that the DSM and in general current diagnostic systems pathologise conditions which are in the range of normality (e.g., Angell, 2011; Cassels, 2013), that the unavailability of biological tests invalidates psychiatric diagnoses (e.g., Roan, 2010; Insel, 2013), and that the Research Domain Criteria (RDoC) project recently launched by the National Institute of Mental Health (NIMH) in the USA (Cuthbert, 2014) is going to transform psychiatric diagnosis by replacing descriptive psychopathology with behavioural and neurobiological measures (e.g., Insel, 2013). In this paper, I briefly discuss these statements and highlight some lessons we can take from this debate.

Let us start from the first statement. Is it true that the DSM is 'the bible of psychiatry', i.e., the main reference for psychiatrists in their diagnostic practice worldwide? An answer to this question can be found in

Address for correspondence: Prof M. Maj, Department of Psychiatry, University of Naples SUN, Largo Madonna delle Grazie, 80138 Naples, Italy.

(Email: majmario@tin.it) the results of a survey recently conducted by the World Psychiatric Association (WPA) and the World Health Organization (WHO) in random representative samples of members of national psychiatric associations of 44 countries (Reed et al. 2011).

From that survey, it emerged first of all that in several countries a substantial minority (or even the majority) of psychiatrists do not use any formal diagnostic system in their ordinary practice. This was reportedly the case, in particular, for more than one-half of the French psychiatrists, more than onethird of the Italian psychiatrists, and more than onequarter of the UK, Australian, Argentinean and Swiss psychiatrists.

Furthermore, when psychiatrists who reported that they did use a formal diagnostic system in their clinical practice were asked to specify which diagnostic system they used, in most countries only a minority stated that they used the DSM. For instance, $<10 \%$ of psychiatrists in Austria, Czech Republic, Finland, Germany, Poland, Russia, Serbia and Switzerland, and slightly more than $10 \%$ of psychiatrists in the UK, France, Brazil and China.

The survey did not explore systematically what the respondents meant when they stated that they used a formal diagnostic system in their ordinary practice. This statement may actually have at least three different meanings: using the labels and/or the codes provided by a diagnostic system, using the diagnostic 
prototypes proposed by a system, or, in the case of the DSM, using the operational diagnostic criteria provided by the system.

Indeed, many European psychiatrists use the ICD-9/10 labels and codes on clinical records because this is required by national regulations, but without having in mind, or sometimes having ever read, the ICD-9/10 text. In other terms, the ICD is often used as a coding rather than a diagnostic system. On the other hand, many psychiatrists use the prototypes of mental disorders delineated by the DSM rather than its operational diagnostic criteria. This has been clearly documented even in the USA by studies carried out in ordinary clinical settings, showing that a large proportion of clinicians have difficulties to memorise and recall, and consequently do not use, even the DSM operational criteria for major depression, which have remained virtually unchanged in the past few decades (e.g., Zimmerman \& Galione, 2010).

From this evidence, we can take our first lesson: that there is a wide gap between current diagnostic systems and ordinary diagnostic practice. We keep on revising those systems, but the impact of these revisions on clinical practice may be much lower than usually realised. This gap will have to be addressed by future research and especially action.

From the WPA-WHO survey, it also emerged that the vast majority of psychiatrists prefer flexible guidance allowing for cultural variation and clinical judgment rather than strict diagnostic criteria. The percentage of psychiatrists expressing this preference was even slightly higher among DSM-IV than ICD-10 users (Reed et al. 2011).

In fact, several recent papers (e.g., Ortigo et al. 2010) have pointed out that the spontaneous clinical process does not involve checking in a patient whether each of a series of symptoms is present or not. It rather involves a matching between the characteristics of the current patient and the templates of the various mental disorders that the clinician has built up in his mind.

In addition to this, it has been repeatedly shown that several of the cutoffs and time frames included in the DSM operational criteria do not have a solid empirical basis. They were originally proposed on the basis of a consensus among experts, in the hope that they would be then validated by empirical evidence, but this evidence in several cases has not become available.

This is the case, for instance, of major depression. Although the operational criteria for that diagnosis have remained virtually unchanged in the past 40 years, empirical research has not supported the thresholds fixed by those criteria for number and duration of symptoms (Kessler et al. 1997; Kendler \& Gardner, 1998).
Furthermore, it has been repeatedly argued that operational criteria, especially for some mental disorders such as schizophrenia, tend to oversimplify psychopathology and may have led to the loss of the psychopathological core of those disorders (e.g., Maj, 1998; Parnas, 2012).

It is commonly held that the introduction of operational criteria has been the most important contribution of the DSM-III, but this view may be incorrect. The most significant contribution may have been the explicit and precise delineation of the prototypes of the various mental disorders, shared by thousands of clinicians in many countries thanks to the wide dissemination that the manual has received, and widely incorporated in the ICD-10, rather than the provision of thresholds in terms of number and duration of symptoms, whose empirical basis remains limited and which are not extensively used in clinical practice, even in the USA.

It is interesting to point out that this was the original vision of Robert Spitzer when the process of development of the DSM-III started in the mid-1970s. In a paper published in 1975, he stated that 'the operational criteria would not replace but just supplement the narrative definitions of the various diagnostic categories... they would be suggested only, and any clinician would be free to use them or ignore them as he saw fit' (Spitzer et al. 1975). Although this prediction did not come true as far as the structure of the DSM-III and its successors is concerned, one could argue that it has largely come true in terms of clinical practice.

It is very telling that several statements in line with the spirit of Spitzer's prediction appear now in the introduction to the DSM-5: for instance, that 'diagnostic criteria are offered as guidelines for making diagnoses, and their use should be informed by clinical judgment', and that 'it is not sufficient to simply check off the symptoms in the diagnostic criteria to make a mental disorder diagnosis' (American Psychiatric Association, 2013).

At the same time as the drawbacks of the operational approach have become more visible, the prototypematching approach to psychiatric diagnosis has regained some ground. Several studies have reported that systems based on detailed narrative descriptions of the prototypes of various mental disorders and requiring the clinician to assess the extent of match of each individual case to each prototype may be as reliable as the DSM operational system in ordinary practice, while being more user friendly and more in line with the spontaneous clinical process (e.g., Westen \& Shedler, 2000; Westen et al. 2012).

Nevertheless, the prototype-matching approach also has its drawbacks (Maj, 2011). Clinicians may have 
problems to memorise and recall the prototypes. They may be reluctant to change the templates of mental disorders they have built up in their mind along the years, or they may adapt, consciously or unconsciously, the proposed prototypes to those templates. Furthermore, the expectation that all the various components of a prototype be present may lead the clinician to infer the presence in an individual patient of clinical aspects which do not actually appear in that patient. Finally, a clinician may conclude that a patient matches a given prototype because several elements of the description are present, while another clinician may conclude that the same patient does not match the prototype because some aspects of the description are absent.

From this evidence and these arguments, we can take the second of our lessons: that we need to assess systematically the pros and cons of the operational $v$. the prototype-matching approaches, with a special focus on their applicability in ordinary practice and their clinical utility. The fact that we have two diagnostic systems in psychiatry, one based on operational criteria and the other on prototypes, may facilitate this comparative effort, which has not been carried out systematically up to now.

Let us proceed now to address the second of the comments made within the above-mentioned campaign, i.e., that the DSM and in general our diagnostic systems pathologise conditions which are in the range of normality.

This critical comment actually has two components (Maj, 2014a). The first is that some of the conditions included in the DSM-5 may not qualify as mental disorders. The second is that the threshold for the diagnosis of some conditions which do qualify as mental disorders may be too low, so that also normal states are included.

To address the first component of the criticism, we should ideally rely on a clear definition of what a mental disorder is, in order to check, when a new condition is proposed for inclusion in the diagnostic system, whether the condition fulfils that definition. Unfortunately, we currently miss that clear definition.

The DSM-5, as its predecessors, does provide a definition of mental disorder in its introduction (American Psychiatric Association, 2013), but that definition is far from being exhaustive and practically useful. In fact, it contains a first element which is tautological (mental disorder is what is clinically significant); a second element which cannot be verified at the present state of knowledge (that there is a dysfunction in the psychological, biological or developmental processes underlying mental functioning); a third element which is present in a variety of conditions that are not necessarily mental disorders (that there is a significant distress or social disability); and a fourth element whose assessment is fraught with a variety of possible confounders (that the condition is not an expectable or culturally approved response to a common stress or loss).

Not surprisingly, several decisions about inclusion or exclusion of proposed new conditions in the DSM-5 have not been guided by that definition, and have been made on the basis of other criteria, applied inconsistently. Let me provide some examples.

The new condition called disruptive mood dysregulation disorder has been included in the system in spite of a test-retest reliability in field trials judged to be 'questionable' (Regier et al. 2013), a research evidence largely coming from a single American group, and an insufficient information about its prevalence in the community and its separation from another condition, oppositional defiant disorder (Leibenluft et al. 2012). The decisive element has been the need to address concerns about overdiagnosis and overtreatment of bipolar disorder in children (American Psychiatric Association, 2013). This pragmatic criterion, perhaps reasonable in the case of the USA, does not hold, however, in an international perspective, because the overdiagnosis and overtreatment of bipolar disorder in children is not occurring in virtually all other countries, where instead the new proposed category, for which no validated treatment is currently available, is likely to generate itself an overdiagnosis of mental disorder and an overuse of psychotropic drugs in children.

The second example regards the attenuated psychosis syndrome, excluded from the main body of the DSM-5 in spite of a good test-retest reliability in field trials (regarded however as inaccurate due to the small sample size) (Regier et al. 2013) and a research evidence emanating from several groups in various countries. In the presence of a situation which was actually less favourable (a good test-retest reliability in field trials, but again regarded as inaccurate due to the small sample size, and a research evidence emanating from just a few groups), another condition, hoarding disorder, has been instead included in the system, perhaps because the balance between the risks and the benefits of the inclusion was regarded as less controversial.

From all this we can take our third lesson: that we need to develop a pragmatic set of inclusion and exclusion criteria to apply explicitly and consistently when the introduction of a new condition in the diagnostic system is proposed. If a balance between the possible benefits of the inclusion in terms of prediction of outcomes and response to available treatments and the possible risks in terms of stigma and inappropriate use of medications is involved in this decision, this will have to be made explicit. 
Let us consider now the second element of the criticism, i.e., that the threshold for the diagnosis of some conditions which do qualify as mental disorders is too low in the DSM.

To address this issue, we must fully acknowledge the reality that most mental disorders lie on a continuum with the range of normality in the general population. This situation is not at all specific to mental disorders. It can be found, in fact, also in several physical conditions, such as hypertension and diabetes (Maj, 2013c). In all conditions in which this continuity between the range of normality and pathology does exist, the boundary between normality and disorder has to be decided arbitrarily on pragmatic grounds, that is, on the basis of clinical utility, especially prediction of outcomes, including response to treatments (Kendell \& Jablensky, 2003).

The problem with the DSM-III and its successors, however, is that several of the thresholds they fix are not only arbitrary, as they are supposed to be, but also not based on solid pragmatic grounds. We have already seen that this is the case for the thresholds fixed for the diagnosis of major depression. Unfortunately, the DSM-5 field trials have ignored this issue. In the case of major depression, the threshold for the diagnosis has been even lowered in the DSM-5 by eliminating the bereavement exclusion criterion, a decision not only not supported but even contradicted by the available research evidence (e.g., Wakefield \& First, 2012; Wakefield, 2013).

From this we can take our fourth lesson: that we cannot keep on using thresholds which are not validated by research just in the name of reliability or in order not to change our current assessment instruments. We need studies formally comparing alternative thresholds for the diagnosis of each mental disorder, especially with respect to their clinical utility. Meanwhile, using clinical judgment to assess the extent of match of each individual case to the prototype of each mental disorder is a reasonable alternative, in ordinary clinical practice, to the use of criteria that are actually pseudo-precise (Maj, 2013b).

Let us proceed now to consider the third comment made in the above-mentioned media campaign: that the unavailability of biological tests invalidates psychiatric diagnoses.

We have to acknowledge first of all that it is not true that psychiatry is unique in the field of medicine in making diagnoses which are not 'based on biological tests'. Migraine and multiple sclerosis are widely quoted examples of physical conditions whose diagnosis remains a clinical one, and hundreds of other diagnoses in medicine have been made correctly for centuries on a clinical basis before laboratory tests became available (Carroll, 2013a).
Furthermore, it is important to emphasise that most laboratory tests in medicine are probabilistic, not pathognomonic, markers of disease. They help in revising diagnostic options rather than conclusively ruling in or out a diagnosis. Their results have always to be interpreted on the basis of clinical judgment (Carroll, 2013b). A paper recently published in the JAMA Internal Medicine actually reported that, in medicine, the patient's history typically accounts for $75 \%$ of more of the diagnostic decision when evaluating common symptoms, while physical examination accounts for $10-15 \%$ and diagnostic tests generally for $<10 \%$ (Kroenke, 2013).

Indeed, the availability of biological tests has not prevented some non-psychiatric diseases which lie on a continuum with normality, such as hypertension and diabetes, to become the subject of controversy as to the appropriate threshold for the diagnosis. In fact, whether blood pressure or glycaemic levels are normal or pathological depends on the clinical outcomes they predict, and the relevant evidence may under some circumstances, for instance during pregnancy for glycaemia, be unclear or controversial, so that the threshold for the diagnosis remains debatable (Ryan, 2011).

From this comes our fifth lesson: that the crucial element is not whether the threshold for the diagnosis of a disorder is based on a biological test or a set of clinical variables, but whether the threshold has a sufficient predictive validity. So, in the absence of biological tests, we should actively search for clinical thresholds that are predictively valid.

This brings us to address the last statement made in the press campaign: that the RDoC approach can transform psychiatric diagnosis in the foreseeable future.

The declared aim of the RDoC project is to generate a diagnostic system based upon neuroscience and behavioural science rather than descriptive phenomenology (Cuthbert, 2014). To pursue this objective, five functional domains have been identified on the basis of a consensus between experts, each consisting of behavioural dimensions that have been at least preliminarily related to a particular brain circuit or area.

The project endorses the assumption that all mental disorders are brain diseases, and can therefore be exhaustively described in terms of abnormalities of brain circuits. This assumption, however, is far from being proved (Maj, 2013a). Damage to the neural substrate may not be necessary for failure of psychological function (e.g., Bolton, 2008). Moreover, a variety of higher-order processes may intervene between the level of neurobiological vulnerability and that of psychopathological manifestations, so that a bottomup reductionistic approach becomes insufficient (Kendler, 2005). Not surprisingly, a major criticism 
which has been addressed to the RDoC project is that it downplays the role of meaning, subjective experience and mental representations in mental disorders (Parnas, 2014; Wakefield, 2014).

The second criticism is a pragmatic one. The main goal of medical, including psychiatric, classification is clinical utility, which is only partly related to underlying pathophysiology (Stein, 2014). To usefully replace current symptom-based diagnostic categories, the proposed new set of behavioural and biological measures will need to have a test-retest and interoperator reliability and a sensitivity and specificity in predicting outcomes at least equal to current symptom-based measures, and to be applicable and cost-effective in a reasonable range of clinical settings (Maj, 2014b; Weinberger \& Goldberg, 2014), something clearly not attainable in the foreseeable future.

The RDoC project is more likely to develop neurobiological measures which help in subtyping rather than in replacing current diagnostic entities, with the aim to improve prediction of outcome and treatment response. So, the lesson is that RDoC supporters should refrain from a polemic confrontation with the DSM and the ICD (e.g., Insel, 2013) which is unwarranted, disruptive to the field, and confusing to patients and families, to policy makers and to the public opinion. On the other hand, it is advisable for clinicians and scholars to keep an open attitude towards the RDoC project. In fact, by interfacing more directly with the level of neuroscience, this project is likely to usefully complement current diagnostic systems, which interface more directly with the level of clinical reality (Maj, 2014b).

Developing cross-walks between the RDoC and the DSM/ICD approaches, in a climate of reciprocal respect, is an endeavour that can only enrich psychiatry and related disciplines. On the clinical side, this may involve the search for experiential, as opposed to behavioural, intermediate phenotypes (e.g., the primary pathological experiences underlying delusions in schizophrenia), the refinement of currently identified dimensions of some disorders, a clearer definition of broader dimensional groupings or spectra (e.g., internalising/externalising disorders), and a greater emphasis on the longitudinal component in the assessment of psychopathology, including the identification of definite stages when possible.

So, in conclusion, there are certainly many problems in clinical diagnosis in psychiatry, and many developments are desirable and feasible. However, trashing current diagnostic practices is clearly unwarranted, in addition to being harmful to the image of our profession and likely to widen the treatment gap that we already face in our field.
Our current diagnostic systems, notwithstanding their limitations, are the outcome of many decades of clinical experience and thousands of research studies. Any new developments will have not only to be intellectually rewarding, but result in something which is more clinically useful - to psychiatrists and especially to service users.

\section{Financial support}

This research received no specific grant from any funding agency, commercial or not-for-profit sectors.

\section{Conflict of interest}

The author declares no conflict of interest.

\section{References}

American Psychiatric Association (2013). Diagnostic and Statistical Manual of Mental Disorders, 5th edn. American Psychiatric Association: Arlington.

Angell M (2011). The epidemic of mental illness: why? The New York Review of Books, June 23.

Bolton D (2008). What is Mental Disorder? An Essay in Philosophy, Science, and Values. Oxford University Press: Oxford.

Carroll BJ (2013a). Quoted in: Frances A. The role of biological tests in psychiatric diagnosis. Huffington Post, May 22.

Carroll BJ (2013b). Biomarkers in DSM-5: lost in translation. Australian and New Zealand Journal of Psychiatry 47, 676-681.

Cassels C (2013). DSM-5 officially launched, but controversy persists. Medscape Multispecialty, May 18.

Cuthbert BN (2014). The RDoC framework: facilitating transition from ICD/DSM to dimensional approaches that integrate neuroscience and psychopathology. World Psychiatry 13, 28-35.

Horgan J (2013). Psychiatry in crisis! Mental health director rejects psychiatric "bible" and replaces with... nothing. Scientific American, May 4.

Insel TR (2013). Transforming diagnosis. NIMH Director's Blog, April 29.

Kendell R, Jablensky A (2003). Distinguishing between the validity and utility of psychiatric diagnoses. American Journal of Psychiatry 160, 4-12.

Kendler KS (2005). Toward a philosophical structure for psychiatry. American Journal of Psychiatry 162, 433-440.

Kendler KS, Gardner Jr CO (1998). Boundaries of major depression: an evaluation of DSM-IV criteria. American Journal of Psychiatry 155, 172-177.

Kessler RC, Zhao S, Blazer DG, Swartz M (1997). Prevalence, correlates, and course of minor depression and major depression in the National Comorbidity Survey. Journal of Affective Disorders 45, 19-30. 
Kroenke K (2013). Diagnostic testing and the illusory reassurance of normal results. JAMA Internal Medicine 173, 416-417.

Leibenluft E, Uher R, Rutter M (2012). Disruptive mood dysregulation with dysphoria disorder: a proposal for ICD-11. World Psychiatry 11(Suppl. 1), 77-81.

Maj M (1998). Critique of the DSM-IV operational diagnostic criteria for schizophrenia. British Journal of Psychiatry 172, 458-460.

Maj M (2011). Psychiatric diagnosis: pros and cons of prototypes vs. operational criteria. World Psychiatry 10, 81-82.

Maj M (2013a). Mental disorders as "brain diseases" and Jaspers' legacy. World Psychiatry 12, 1-3.

Maj M (2013b). "Clinical judgment" and the DSM-5 diagnosis of major depression. World Psychiatry 12, 89-91.

Maj M (2013c). Adherence to psychiatric treatments and the public image of psychiatry. World Psychiatry 12, 185-186.

Maj M (2014a). DSM-5, ICD-11 and 'pathologization of normal conditions'. Australian and New Zealand Journal of Psychiatry 48, 193-194.

Maj M (2014b). Keeping an open attitude towards the RDoC project. World Psychiatry 13, 1-3.

Ortigo KM, Bradley B, Westen D (2010). An empirically based prototype diagnostic system for DSM-V and ICD-11. In: Contemporary Directions in Psychopathology: Scientific Foundations of the DSM-V and ICD-11 (ed. T Millon, RF Krueger and E Simonsen), pp. 374-390. Guilford: New York.

Parnas J (2012). The core Gestalt of schizophrenia. World Psychiatry 11, 67-69.

Parnas J (2014). The RDoC program: psychiatry without psyche? World Psychiatry 13, 46-7.

Reed GM, Mendonça Correia J, Esparza P, Saxena S, Maj M (2011). The WPA-WHO global survey of psychiatrists' attitudes towards mental disorders classification. World Psychiatry 10, 118-131.
Regier DA, Narrow WE, Clarke DE, Kraemer HC, Kuramoto SJ, Kuhl EA, Kupfer DJ (2013). DSM-5 field trials in the United States and Canada, Part II: test-retest reliability of selected categorical diagnoses. American Journal of Psychiatry 170, 59-70.

Roan S (2010). Revising the book on mental illness. Los Angeles Times, February 10.

Ryan E (2011). Diagnosing gestational diabetes. Diabetologia 54, 480-486.

Spitzer RL, Endicott J, Robins E (1975). Clinical criteria for psychiatric diagnosis and DSM-III. American Journal of Psychiatry 132, 1187-1192.

Stein DJ (2014). An integrative approach to psychiatric diagnosis and research. World Psychiatry 13, 51-53.

Wakefield JC (2013). DSM-5 grief scorecard: assessment and outcomes of proposals to pathologize grief. World Psychiatry 12, 171-173.

Wakefield JC (2014). Wittgenstein's nightmare: why the RDoC grid needs a conceptual dimension. World Psychiatry 13, 38-40.

Wakefield JC, First MB (2012). Validity of the bereavement exclusion to major depression: does the empirical evidence support the proposal to eliminate the exclusion in DSM-5? World Psychiatry 11, 3-10.

Weinberger DR, Goldberg TE (2014). RDoCs redux. World Psychiatry 13, 36-38.

Westen D, Shedler J (2000). A prototype matching approach to personality disorders: toward DSM-V. Journal of Personality Disorders 14, 109-126.

Westen D, Malone JC, DeFife JA (2012). An empirically derived approach to the classification and diagnosis of mood disorders. World Psychiatry 11, 172-180.

Zimmerman M, Galione J (2010). Psychiatrists' and nonpsychiatrist physicians' reported use of the DSM-IV criteria for major depressive disorder. Journal of Clinical Psychiatry 71, 235-238. 